\title{
Parameters Characterization of Dielectric Materials Samples in Microwave and Millimeter-Wave Bands
}

\author{
Mario Pérez-Escribano, Student Member, IEEE, and Enrique Márquez-Segura, Senior Member, IEEE
}

\begin{abstract}
In this work, an optimized broadband method using multilayer transmission lines to characterize dielectric permittivity and loss tangent of material samples is presented. For this purpose, a microstrip line loaded with a piece of the selected dielectric to be characterized is used. From two-port measurements, and using different length lines, the propagation constant can be obtained. To minimize random errors and to improve the accuracy, an over determination of the method increasing the number of lines measured and a criteria to choose the optimal line lengths is considered. Firstly, the measurement method itself is applied to uncovered microstrip lines and an accurate model of the substrate is obtained. Secondly, the lines are covered with several materials, made by FDM additive manufacturing technique, such as Acrylonitrile Butadiene Styrene (ABS), Polylactic Acid (PLA), High Impact Polystyrene (HIPS), Thermoplastic Polyurethane (TPU), Copolyester (CPE), FLEX, Polyethylene Terephthalate Glycol (PETG) and Nylon. A model of the transmission line considering the cover is developed and an electromagnetic simulator is used to indirectly determine the cover material electrical parameters. Results show excellent agreement with electromagnetic simulations in the 0.1- to 67GHz frequency band, so they assess the suitability of the proposed method.
\end{abstract}

Index Terms-broadband measurements, dielectric materials, EM material characterization, propagation constant, transmission line measurements

\section{INTRODUCTION}

$\mathbf{N}$ OWADAYS, the number of materials and manufacturing techniques are available on the market is increasing rapidly. Concretely, additive manufacturing technologies have become one of the most researched topic in many scientific fields. Their main advantages includes the low cost, the possibility of great customization, the rapid prototyping, the design flexibility or the possibility of combining materials. Concretely, in microwave area, several designs have been proposed for different purposes [1]-[4]. Mechanical properties are usually available from manufacturers. However, despite the large amount of materials available, there is not much information about the electromagnetic properties of these materials. Furthermore, the wide variety of manufacturers and materials available make it very necessary to achieve characterization techniques as simple as possible, so that they can be accessible to all designers who want to use this kind of

This work has been supported by the Spanish Ministerio de Economía, Industria y Competitividad under Project ADDMATE TEC2016-76070-C3-3$\mathrm{R}$ (AEI/FEDER, UE) and by the Spanish Ministerio de Educación, Cultura y Deporte under Grant FPU16/00246.

M. Pérez-Escribano and E. Márquez-Segura are with the Departamento de Ingeniería de Comunicaciones, Escuela Técnica Superior de Ingeniería de Telecomunicación, Universidad de Málaga, 29071, Málaga, Spain (e-mail: mpe@ic.uma.es; ems@ic.uma.es). materials. Manufacturers of additive manufacturing materials from time to time change the formulation of materials or even different batches can produce different electromagnetic properties. For those reasons and considering the advantages and growth of this manufacturing technology, investigations on printed electromagnetic material properties have grown in the last few years [5]. Dissipation factor and dielectric permitivitty is determined in an extraordinary band width, for $100 \mathrm{MHz}$ up to the limit of the vector network analyzer available, 67 $\mathrm{GHz}$.

Over time, numerous papers have been proposed in the literature to characterize the propagation constant of planar transmission lines. They can be grouped mainly in two different categories. On the one hand, resonant methods [6], [7] allow to obtain very accurate results and good losses estimation, apart from showing greater independence to the effects caused by transitions. However, they just provide values at the resonant frequencies, so they can not be useful when broadband characterization is required. Several of these methods have been proposed for the characterization of printed materials [8], [9]. On the other hand, broadband methods [10], [11] provide continuous results within the whole frequency band, because they rely on the propagation of the transmission line modes. Nevertheless, their accuracy depends on the precision of the experiments and they show a worst behavior precisely at resonances. In general, broadband techniques extract the propagation constant from the measurements of parameter matrices of transmission lines with different lengths. They have a significant drawback when losses in the transmission medium under test are low, because the attenuation constant can be hidden by noise and random errors. Several broadband methods have been used in microwave [12] and millimeter bands [13] to characterize printed materials. Another novel strategy for the characterization of materials is the one proposed in [14], based on microstrip lines of variable width. It is noteworthy that most of these methods need calibrated $S$ parameters. On the other hand, in this paper the method used do not require calibration due to it is based in mathematical invariant. Some others method, such as those based on Nicolson-RossWier method, do require calibration of the S-parameters to eliminate systematic errors [15], [16]. In order to increase the performance of measurement methods, the overdetermination of samples needed is usually considered [17]-[19].

It is important to mention that these materials under consideration in this paper do not replace conventional low loss materials necessary especially an millimeter band as substrate for microstrip antenna for instance. However, these materials can be used to generate structures in prototyping stages and fi- 


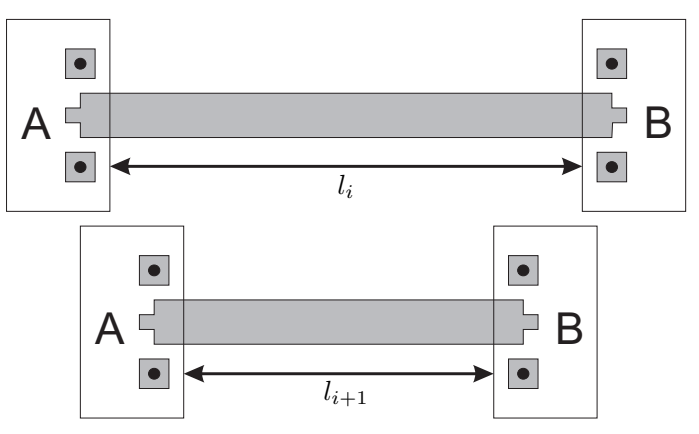

(a)

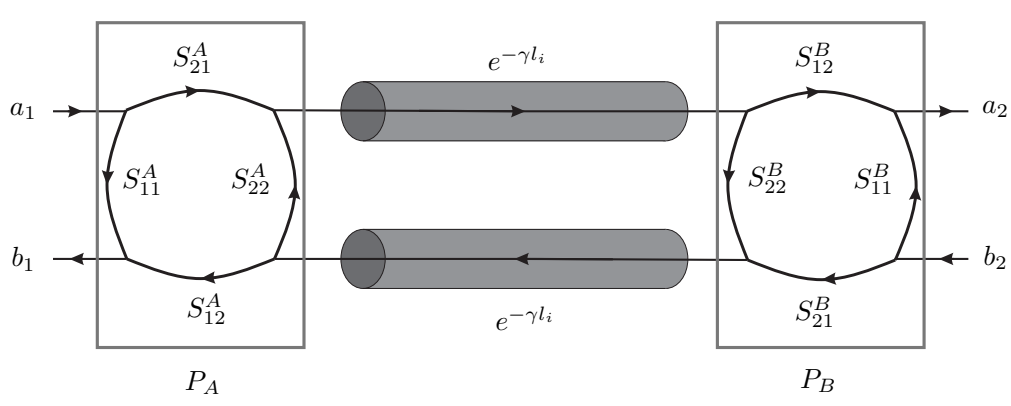

(b)

Fig. 1. (a) Measurement setup using two microstrip lines. (b) Signal-flow of the model including error two-port circuits.

nal products, as cases or structural parts for support for several devices as radars or sensors at microwave or millimeter-wave band. The dielectric parameters should be known accurately to be considered properly in electromagnetic simulator before fabrication. These materials can also play a functional role as ABS in [20], [21] or HIPS in [22]. Particularly, HIPS as a polystyrene variant has demonstrate to be a candidate for millimeter wave material. Other material with flexibility mechanical properties could be useful in microwave band for wearable and conformable circuitry.

In this article, a method to find electromagnetic properties of material samples, made by additive manufacturing FDM, is proposed. It is based on two-port measurements of microstrip transmission lines. For this purpose, it is required the use of a two-port vector network analyzer (VNA) with no need of calibration. The broadband measurement method is described in Section II. In order to achieve a better accuracy of the method, several line length differences are considered and a previous microstrip characterization is carried out. A piece of the material to be characterized is placed over the microstrip line. The extraction of material parameters of a multilayer microstrip is based on the one in [23]. This procedure is shown in Section III. Section IV includes experimental and simulated results for the dielectric permittivity and the loss tangent of the used material samples. Finally, conclusions are provided in Section V.

\section{BROADBAND MEASUREMENT METHOD}

\section{A. Bianco and Parodi two lines method}

The method addressed in this work is based on the one in [10]. This method was then use in the estimation of the propagation constant in TRL calibration for six port transmission measurements and vector network analyzers [24]-[27]. The measurement setup and the signal flow is depicted in Fig. 1. Firstly, it is necessary to take the S-parameters of two lines under test (LUT) with different lengths. It is noteworthy that the error two-port circuits $P_{A}$ and $P_{B}$ are the same throughout the whole set of measurements. The S-parameters have to be transform into T-parameters, that will be called $T_{1}$ and $T_{2}$. These matrices can be rewritten as $T_{1}=P_{A} L_{1} P_{B}$ and $T_{2}=P_{A} L_{2} P_{B}$, being $L_{1}$ and $L_{2}$ the transmission matrices of the lines excluding the effect provoked by the transitions between the LUT and the ports, whose effects are considered in $P_{A}$ and $P_{B}$ respectively. On the one hand, $L_{i}$ are diagonal matrices, given by

$$
L_{i}=\left[\begin{array}{cc}
e^{-\gamma l_{i}} & 0 \\
0 & e^{+\gamma l_{i}}
\end{array}\right],
$$

being $L=L_{1} L_{2}^{-1}$ and $l_{i}$ the length of the line $i$.

On the other hand, the transmission matrices can be combined as

$$
T=T_{1} T_{2}^{-1}=P_{A} L_{1} L_{2}^{-1} P_{A}^{-1}=P_{A} L P_{A}^{-1} .
$$

At this point, it is important to highlight that both $T$ and $L$ are similar matrices [28], which means that their traces and determinants coincide, and their value is given by

$$
\operatorname{trace}(T)=\operatorname{trace}(L)=e^{-\gamma \Delta l}+e^{+\gamma \Delta l},
$$

where $\Delta l=l_{2}-l_{1}$. From (3), the propagation constant can be obtained as

$$
\gamma=\frac{1}{\Delta l} \cosh ^{-1}\left(\frac{\operatorname{trace}(T)}{2}\right) .
$$

Considering Eq. (2), the use of calibrated or raw $\mathrm{S}$ parameters do not affect the results. The inclusion of a new two-port error box cascaded with $P_{A}$ and $P_{B}$, do not affect similarity between the new $T$ and $L$ matrices. Therefore, it is not necessary to eliminate systematic errors calibrating the vector network analyzer before the measurement of transmission lines.

\section{B. Problems due to the existence of multiple solutions}

A problem associated with Eq. (4) is that the inverse hyperbolic cosine has an ambiguity when $\beta \Delta l>\pi / 2$, and phase shifts appear. This is due to the infinite solutions of the function $\cosh ^{-1} z_{g}$ when $z_{g}$ is a complex number. The inverse hyperbolic cosine of a complex number is calculated as

$$
\cosh ^{-1}\left(z_{g}\right)=\ln \left(z_{g}+\sqrt{\left(z_{g}+1\right)\left(z_{g}-1\right)}\right)
$$

As the natural logarithm of a complex number has infinite solutions,

$$
\ln \left(z_{p}\right)=\ln \left|z_{p}\right|+j\left(\arg \left(z_{p}\right)+2 k \pi\right) \quad k=0,1, \ldots,
$$

infinite number of propagation constants are obtained from Eq. (4). 


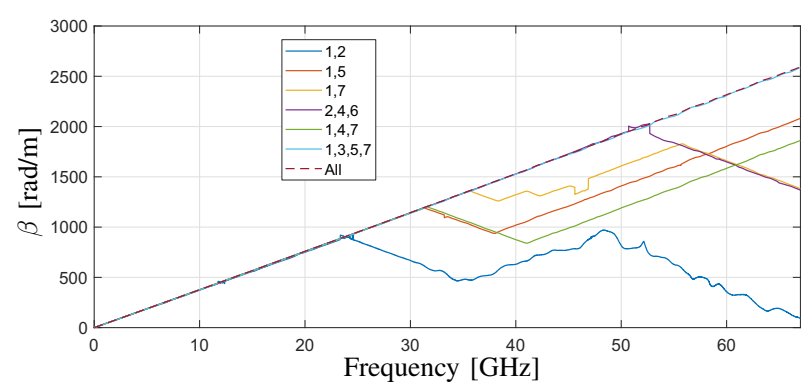

Fig. 2. Phase constants using different line combinations.

In order to fix this problem, Eq. (4) is solved using an iterative technique such as Newton's method to find the complex zero of

$$
\gamma-\frac{1}{\Delta l} \cosh ^{-1}\left(\frac{\operatorname{trace}(T)}{2}\right)=0 .
$$

Nevertheless, this has an inconvenience: to achieve a good method performance, it is necessary to have good starting values, and it is not always possible to get the values that allow to find the correct solution. Starting from the solution of the propagation constant at the lower frequency point, this value is used to initialize the second frequency point and so to the last frequency points. This way, the iterative process starts from a very close point to the correct solution.

\section{Over determination of the method}

Despite the problems associated with multiple solutions of Eq. (4), random errors introduced during the measurements of the $\mathrm{S}$ parameters must be taken into account. Systematic errors could be removed by calibration, although it is not strictly necessary, because port effects are being considered in 4 , by considering that they are going to be the same in all the measurements. For this reason, all experiments performed in this work have been carried out without calibration, i.e. using raw data from the vector network analyzer.

Random errors, however, affect all the measurements and are not predictable or removable by calibration. They can be produced, in part, by the network analyzer. Nevertheless, the most important sources of random errors in the proposed method are the repeatability of the transitions and the estimation of the line lengths used. These errors mean that it is not possible to determine the exact value of the propagation constant, so the objective is to achieve the best estimation from the available measurements.

To improve the accuracy of the method, it is proposed to increase the number of lines to be measured and use a least squares approximation. Thereby, the effects produced by random errors of measurements repeatability are minimized. The propagation constant is obtained by solving the equation

$$
\min _{\alpha, \beta} \frac{1}{2} \sum_{n=1}^{N}\left|\operatorname{trace}(T)-\left(e^{+\gamma \Delta l}+e^{-\gamma \Delta l}\right)\right|^{2},
$$

where $N$ is the number of possible combinations between all the $k$ lines, taken two by two. Increasing $k$ is a good way to reduce uncertainty, but this increment should be accompanied by different $\Delta l$ values, in order to achieve better results. Optimal line lengths selection will be explained in the next section.

Finally, it should be borne in mind that attenuation and phase constants will have very different order of magnitude. For this reason, errors in numerical optimization will penalize $\alpha$ much more than $\beta$. To avoid this, the transformation $\varepsilon_{\mathrm{r}, \mathrm{eff}}=(c \beta / \omega)^{2}$ is applied in order to make both variables have the same order of magnitude, and Eq. (8) is expressed as

$$
\min _{\alpha, \varepsilon_{\mathrm{r}, \mathrm{eff}}} \frac{1}{2} \sum_{n=1}^{N}\left|\operatorname{trace}(T)-\left(e^{+\gamma_{t} \Delta l}+e^{-\gamma_{t} \Delta l}\right)\right|^{2},
$$

being $\gamma_{t}=\alpha+j \omega \sqrt{\varepsilon_{\mathrm{r}, \mathrm{eff}}} / c$.

In order to demonstrate how the method works, Fig. 2 shows the phase constant obtained from measurements of 7 lines with HIPS as the top substrate. The measurement setup will be explained in the next sections, but what is important to note at this point is that phase shifts [29], [30] occur at higher frequencies as the difference in line lengths is greater. From 4 lines the bandwidth of the method reaches the measurement bandwidth. Furthermore, random errors decrease as the number of lines increases.

\section{Optimal line lengths}

Random errors are inherent to any estimation problem based on measurements made by real instruments. Considering equation (4) the attenuation and phase constant are obtained from the measurement of different lengths $\Delta l_{k}$ and the uncalibrated $S$ parameters measured using a vector network analyzer. In order to minimize the effect of these random errors, special care must be taken in connections repeatability, similarity of connectors and length of lines measurement.

Over determination of the method proposed using more than two lines will help to minimize errors significantly. The measurements of $\mathrm{S}$ parameters of $N$ lines, $N>2$, get to $\mathrm{M}$ simultaneous non-linear complex equation. The value of $M$ is given by the combinations of $N$ lines taken 2 at a time: $M=1 / 2 \cdot N(N-1)$. Increasing slightly the number of lines, the total number of combinations increase rapidly and so, the number of estimations of the propagation constant is also increased. In the experiment that has been carried out a number of seven lines provides 21 combinations of two lines.

Apart from a very good statistical data processing and the repeatability of the connections some other considerations must be taken into account to get good estimations of the propagation constant. One of those is the choice of the lengths of the lines employed. The length distribution must be chosen so that the differences between the increments in the length of the lines should be as small as possible. In addition, these increments must be the bigger the better. In this way, the experiments to determine the propagation constant are more insensitive to the relative errors in the determination of the lengths.

Constant increments in length between different transmission lines concentrates resonances in discrete number of points. Small deviations from that pattern spread the resonances through the band of interest. The selected lengths 


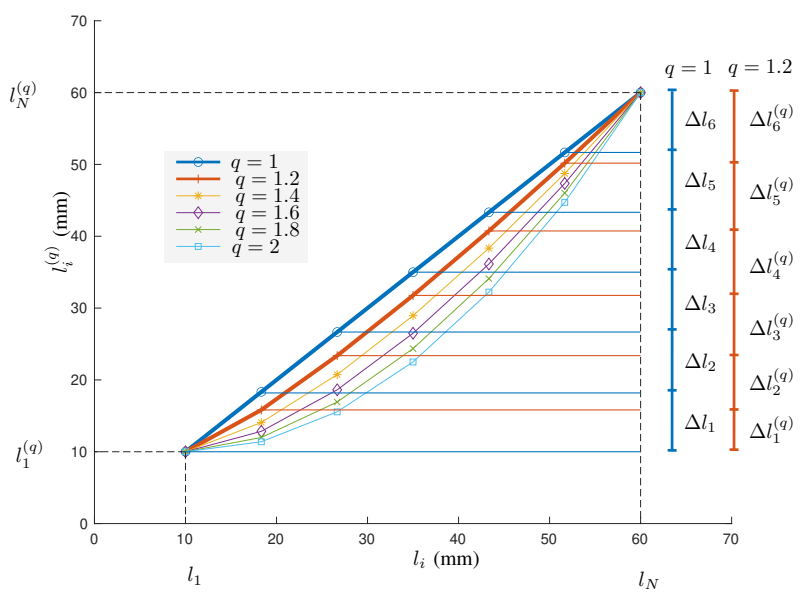

(a)
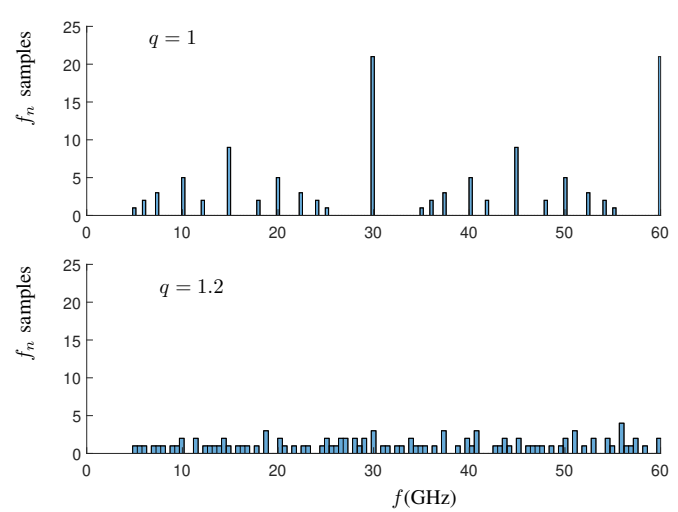

(b)

Fig. 3. (a) Quasi-linear lengths of lines under test. (b) Histograms of $f_{n}$ for different values of factor $q$.

follow a quasi-linear distribution as shown in the following equation

$$
l_{i}=l_{0}+\left(l_{N}-l_{0}\right)\left(\frac{i-1}{N-1}\right)^{q}, i=1,2, \ldots N,
$$

where $l_{0}$ is the shortest line length and $l_{N}$ is the longest. The quasi-linear factor $q$ just need to be adjust to a value different of $1, q=1.2$ has empirically demonstrated that the distribution of resonances spread enough from a practical point of view.

The frequencies where the phase difference of measured $S_{21}$ is zero can be easily obtained as

$$
f_{n}=\frac{n \cdot c}{\left(l_{b}-l_{a}\right) \sqrt{\varepsilon_{\mathrm{r}, \mathrm{eff}}}} .
$$

Figure 3 shows the difference in the increment $\Delta l_{i j}$ for different strategies. Fig. 3.b compares histograms where the aggregation of frequencies where the phase difference between measured $S_{21}$ appears. For $q=1.2$ the distribution is homogeneous through the frequency band.

\section{E. Microstrip characterization}

Once the method has been presented, it is evaluated through experimental validation and electromagnetic simulation. For this, 7 microstrip lines are manufactured over Rogers
4350B LoPro substrate, with 30 mil thickness, $\varepsilon_{\mathrm{r}}=3.66$, $\tan \delta=0.0031$, and $17.5 \mu \mathrm{m}$ thick copper metallization. The length of the lines, shown in Fig. 4 caption, is calculated following the procedure explained in the previous section. The line width is set to $1.65 \mathrm{~mm}$, in order to get a $50 \Omega$ characteristic impedance.

The characterization method consists of measuring the Sparameters of the 7 lines. Later, Eq. (9) is applied, in order to obtain $\alpha$ and $\varepsilon_{\mathrm{r}, \text { eff }}$, from which the estimated nominal $\varepsilon_{\mathrm{r}}$ of the substrate, including dispersion, is extracted using the Kirschning and Jansen model [31]. Finally, the obtained $\varepsilon_{\mathrm{r}}$ is imported in the commercial full-wave finite-element electromagnetic simulator ANSYS HFSS and one of the 7 microstrip lines is simulated, getting its propagation constant.

Figure 4 shows the measured and simulated effective permittivity and the attenuation constant obtained through the method application to the set of 7 lines. In addition, the information about the substrate dispersion given by the material manufacturer [32] up to $50 \mathrm{GHz}$ is also depicted. As seen, there is an excellent agreement between the measured effective relative permittivity and the one obtained by simulation using manufacturer data. In this sense, it must be highlighted that the relative permittivity, obtained from the one in Fig. 4.b using the Kirschning and Jansen model and plotted in Fig. 4.c, is quite similar to the one given by Rogers, showing how good is the proposed method for estimating this parameter. For this reason, the obtained $\varepsilon_{\mathrm{r}}$ of the Rogers 4350B LoPro substrate will be used in the next section for the simulation of the proposed structure in order to characterize more precisely, including lower substrate dispersion, the material samples.

\section{ESTIMATION OF MATERIAL PARAMETERS}

The setup proposed for the estimation of the dielectric parameters consists of a microstrip line loaded with a dielectric overlay, made of the material that will be characterized. The measurement scheme is shown in Fig. 5. As shown, microstrip dielectric has a known permittivity $\varepsilon_{\mathrm{r} 1}$, estimated in the previous section, and a height $h_{1}$, whereas the overlay has an unknown permittivity $\varepsilon_{\mathrm{r} 2}$ and a height $h_{2}$.

The procedure consists of the determination of the propagation constant of the loaded microstrip, and then, the extraction of the attenuation and phase constant of the dielectric overlay. Several numerical models has been proposed to estimate the effective relative permittivity of this structure, highlighting [23], [33]. Both models are based on numerical methods, and come from [34].

The problem with these methods is that the estimated relative permittivity is calculated using a quasi-static model. For that reason, they do not take into account the material's own dispersion. As seen in Fig. 4, relative permittivity has a slight downward trend with frequency, and this trend will also appear in printing materials. For that reason, a mathematical model that characterizes this decreasing curve between the minimum and the maximum frequencies that our analyzer allows us to measure will be raised. This curve model will be defined as

$$
\varepsilon_{\mathrm{ri}}=\varepsilon_{\mathrm{r} 1}-\left(\varepsilon_{\mathrm{r} 67}-\varepsilon_{\mathrm{r} 1}\right)\left(\frac{i-1}{N-1}\right)^{p}, i=1, N,
$$




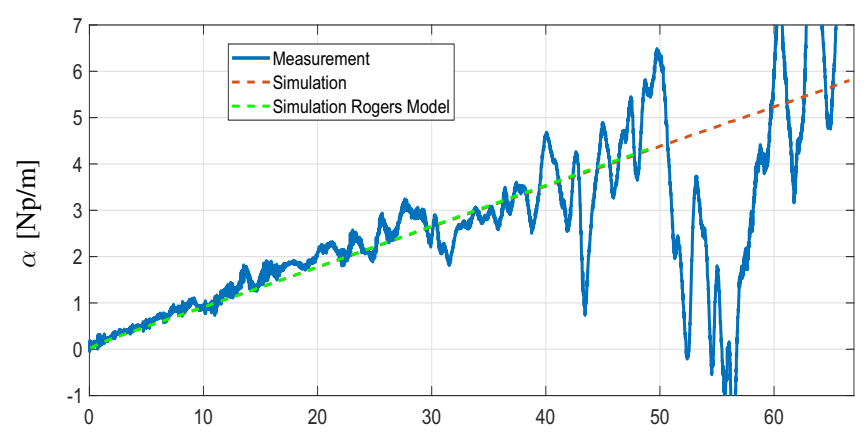

(a)

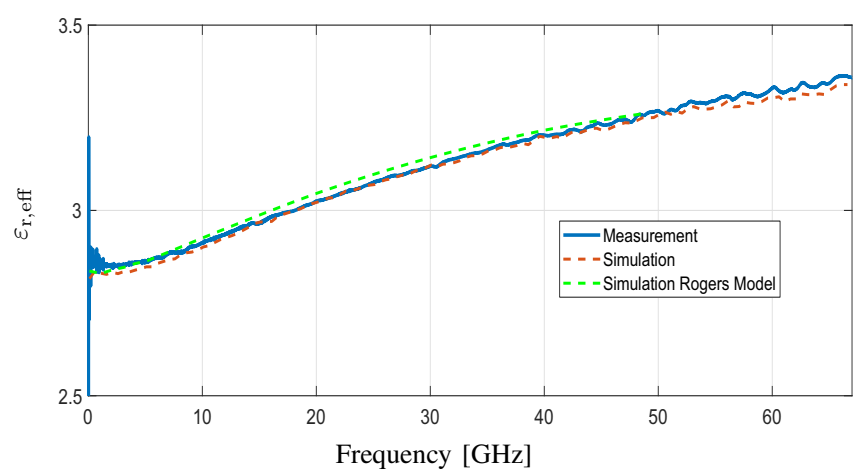

(b)

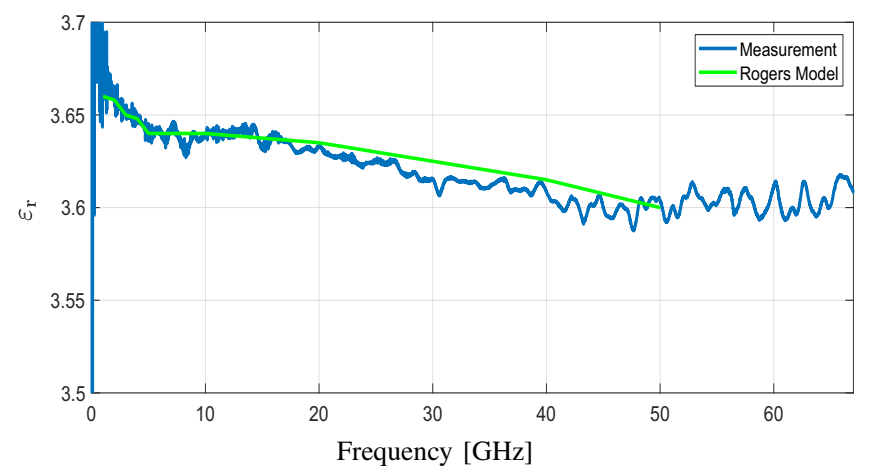

(c)

Fig. 4. Experimental results of the propagation constant using the proposed method with 7 line lengths in comparison with electromagnetic simulation results. The line lengths are 2, 2.69, 3.60, 4.61, 5.68, 6.82 and $8 \mathrm{~cm}$. (a) Attenuation constant. (b) Relative effective permittivity. (c) Estimated substrate permittivity.

where $\varepsilon_{\mathrm{r} 1}$ and $\varepsilon_{\mathrm{r} 67}$ are the estimated relative permittivity at 1 $\mathrm{GHz}$ and $67 \mathrm{GHz}$ respectively, and $p$ is a quasi-linear factor. This value can be $p=1$ for a linear model, $p=2$ for a quadratic model or a value between these values, that will suppose a quasi-linear model. After comparing measurements and simulations, the value $p=1.5$ shows that minimal differences are achieved, and, therefore, it is selected for this model.

To determine losses, the process will consist of two parts. On the one hand, it will be necessary to determine losses in conductors. For this process, the analytical model presented in [34] and improved in [35], which includes the effect of surface roughness, will be used. Once these losses have been

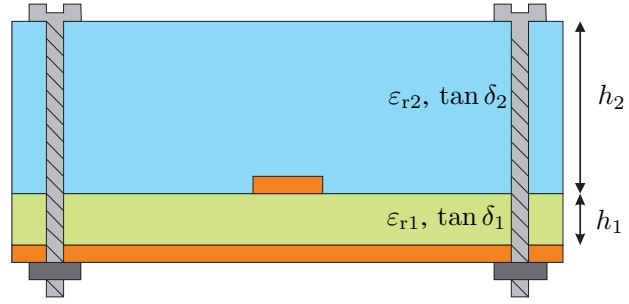

Fig. 5. Covered microstrip lines using nylon screws for layers bonding.

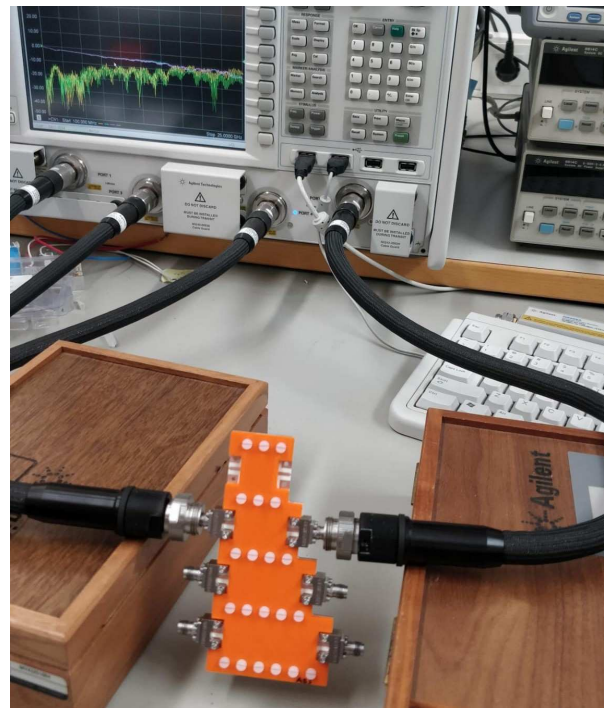

Fig. 6. Photograph of microstrip lines covered by orange ABS and the measurement setup using vector network analyser for S-parameters measurement.

considered, it is necessary to determine losses in dielectrics. This process is based on finding the $\tan \delta_{e q}$ of both dielectrics. This equivalent model was defined in [36]. From it, knowing the loss tangent of the lower substrate $\tan \delta_{1}$, it is possible to obtain the loss tangent of the upper substrate $\tan \delta_{2}$.

\section{RESULTS}

To validate the developed method, a sample of RO4350B has been made in order to use it as top substrate. As it is a material whose permittivity is known, it will be used to assess the method performance. In addition, pieces of HIPS, 2 different ABS, TPU, CPE, NYLON, FLEX, PLA and PETG have been manufactured and measured. In the printing process, a $100 \%$ infill has been used, in order to estimate the pure material characteristics. If a smaller infill had been used, lower permittivities and losses would have been obtained [12], because there would be air inside the substrate. However, in this point it is important to remark that not all printers manufacture in the same way or following the same patterns a $100 \%$ infill, and probably there will always be a small amount of air between the sheets of the substrate. For that reason, printer and printing setup should be specified when characterizing materials.

A photograph of the manufactured structure is shown in Fig. 6. In that Figure, it is possible to see the uncalibrated measured data on the VNA screen. The junction between the 
TABLE I

COMPARISON BETWEEN THE ANALYZED MATERIALS

\begin{tabular}{ccccccccc} 
Material & $\varepsilon_{\mathrm{r}} @ 1 \mathrm{GHz}$ & $\varepsilon_{\mathrm{r}} @ 10 \mathrm{GHz}$ & $\varepsilon_{\mathrm{r}} @ 25 \mathrm{GHz}$ & $\varepsilon_{\mathrm{r}} @ 67 \mathrm{GHz}$ & $\tan \delta$ & $\alpha @ 1 G H z[\mathrm{~dB} / \mathrm{m}]$ & $\Delta_{1}$ & $\Delta_{2}$ \\
\hline Rogers 4350B LoPro & 3.638 & 3.611 & 3.592 & 3.578 & 0.0039 & 0.67 & $12.1 \times 10^{-3}$ & $6.4 \times 10^{-3}$ \\
\hline HIPS & 2.45 & 2.438 & 2.424 & 2.4 & 0.004 & 0.57 & $5.2 \times 10^{-3}$ & $3.2 \times 10^{-3}$ \\
ABS* & 2.67 & 2.655 & 2.638 & 2.61 & 0.009 & 1.34 & $6.9 \times 10^{-3}$ & $2.1 \times 10^{-3}$ \\
TPU & 2.67 & 2.645 & 2.618 & 2.57 & 0.032 & 4.75 & $16.7 \times 10^{-3}$ & $6.4 \times 10^{-3}$ \\
CPE & 2.8 & 2.788 & 2.774 & 2.75 & 0.013 & 1.98 & $4.9 \times 10^{-3}$ & $2.2 \times 10^{-3}$ \\
NYLON & 2.95 & 2.94 & 2.929 & 2.91 & 0.019 & 2.97 & $11.6 \times 10^{-3}$ & $5.6 \times 10^{-3}$ \\
ABS2 & 2.7 & 2.683 & 2.663 & 2.63 & 0.009 & 1.35 & $6.9 \times 10^{-3}$ & $2.1 \times 10^{-3}$ \\
FLEX & 2.65 & 2.633 & 2.613 & 2.58 & 0.036 & 5.33 & $5.2 \times 10^{-3}$ & $3.2 \times 10^{-3}$ \\
PLA & 2.5 & 2.483 & 2.463 & 2.43 & 0.013 & 1.87 & $5.3 \times 10^{-3}$ & $2.4 \times 10^{-3}$ \\
PETG & 2.85 & 2.83 & 2.808 & 2.77 & 0.022 & 3.38 & $7.2 \times 10^{-3}$ & $3.1 \times 10^{-3}$
\end{tabular}

TABLE II

PRINTING SETUP OF EACH MATERIAL

\begin{tabular}{ccccccc} 
Material & Manufacturer & Printer & Filament Size $(\mathrm{mm})$ & Infill $(\%)$ & Extrusion temperature $\left({ }^{\circ} \mathrm{C}\right)$ & Bed temperature $\left({ }^{\circ} \mathrm{C}\right)$ \\
\hline HIPS & Orbi-Tech & Ultimaker 3 & 2.85 & 100 & 235 & 100 \\
ABS* & Ultimaker & Ultimaker 3 & 2.85 & 100 & 250 & 90 \\
TPU & Ultimaker & Ultimaker 3 & 2.85 & 100 & 225 & 70 \\
CPE & Ultimaker & Ultimaker 3 & 2.85 & 100 & 245 & 75 \\
NYLON & Ultimaker & Ultimaker 3 & 2.85 & 100 & 240 & 70 \\
ABS2* & Smartmaterials3D & Prusa i3 MK3 & 1.75 & 100 & 240 & 50 \\
FLEX & Smartmaterials3D & Prusa i3 MK3 & 1.75 & 100 & 240 & 50 \\
PLA & Smartmaterials3D & Prusa i3 MK3 & 1.75 & 100 & 215 & 75 \\
PETG & Smartmaterials3D & Prusa i3 MK3 & 1.75 & 100 & 235 &
\end{tabular}

ABS* and ABS2*: These materials were manufactured with the printer fully covered to avoid air flows that could cause delaminations.

substrate and the manufactured piece is made with screws. In order to ensure the repeatability of the transitions and minimize errors in cable movement, the same 2 connectors were used for measuring all the lines, changing them for each measurement. Furthermore, a torque wrench was used to ensure that all connectors are equally tight, and a visual inspection with microscope was made before each measurement. Lastly, a rigid plate was placed under the substrate to ensure that it remains completely straight during every measurement.

The microstrip line, as well as the length of the lines, are the same that the one used during the microstrip characterization in section II.E. S-parameters of the 7 lines are taken by using the network analyzer Agilent PNA-X (N5247A), between 0.1 and $67 \mathrm{GHz}$, that is the maximum capability of that analyzer. Using the electromagnetic simulator ANSYS HFSS, a structure as shown in Fig. 5 has been simulated for every material. A $0.4 \mu \mathrm{m}$ surface roughness [37], considering the Groisse model [38], has been used for the simulations. The heights of the substrates are $h_{1}=0.762 \mathrm{~mm}$ and $h_{2}=3 \mathrm{~mm}$, respectively. The gap between the substrates will cause the obtained relative permittivity to vary slightly. For this reason, some simulations have been carried out in order to assess this variation, using the maximum gap, which is the height of the metallization $(17 \mu \mathrm{m})$, and the minimum gap $(0 \mu \mathrm{m})$. The gap works as a bias in the relative permitivitty. However, the maximum difference between $\varepsilon_{\mathrm{r} 2}$ values obtained with the aforementioned gaps is $0.35 \%$, which means that the error made by the separation between substrates is very small. The dielectric permittivity of the Rogers 4350B LoPro lower substrate, used in the simulation $\left(\varepsilon_{\mathrm{r} 1}\right)$ is the one shown in Fig. 4.c, that was calculated by using the proposed method. The dielectric permittivity of the printed material $\left(\varepsilon_{r 2}\right)$ for the simulation is calculated from the measured results, considering the dispersion model raised in Eq.(12). Specifically, the values of $\varepsilon_{\mathrm{r}}$ of the Rogers top substrate and the material samples at $1,10,25$ and $67 \mathrm{GHz}$, as well as the $\tan \delta$ used for the simulations and the attenuation constant at $1 \mathrm{GHz}$ for a plane wave are depicted in Table I. As seen, results for the Rogers material are quite near to the one given by the manufacturer, shown in Fig. 4.c. Small differences, that are less than $1 \%$, are probably due to the small gap between both substrates or permittivity temperature dependence. Regarding printed materials, results are also similar to those found in other works [39]-[41], even considering that printing materials are not completely pure materials. Despite the variety of the materials studied, they all show a relative permittivity between 2.45 and 2.95. This fact is because they are all plastic materials and manufactured with the same manufacturing process. However, there are significant differences in terms of loss tangent, which makes some materials clearly candidates for use for microwave circuits (i.e., HIPS or ABS), while others are not as suitable (i.e., flexible materials such as TPU or FLEX).

In order to guarantee the repeatability of the process, the printing setup, including the manufacturer of each material, the printer used, the filament size, the infill, the extrusion temperature and the bed temperature are shown in Table II. In order to validate the performance of the dispersion model in Eq.(12), the operator $\Delta$ is defined as

$$
\Delta=\frac{1}{N} \sum_{i=1}^{N}\left|\varepsilon_{\mathrm{r}, \mathrm{eff}_{i}}^{\mathrm{sim}}-\varepsilon_{\mathrm{r}, \mathrm{eff}_{i}}^{\text {meas. }}\right|^{2},
$$

where $N$ is the number of points of the measurement and simulation. In Table I, $\Delta_{1}$ is obtained by using a simulation where dispersion is not considered, while $\Delta_{2}$ is obtained by using the raised dispersion model. As seen, lower differences 


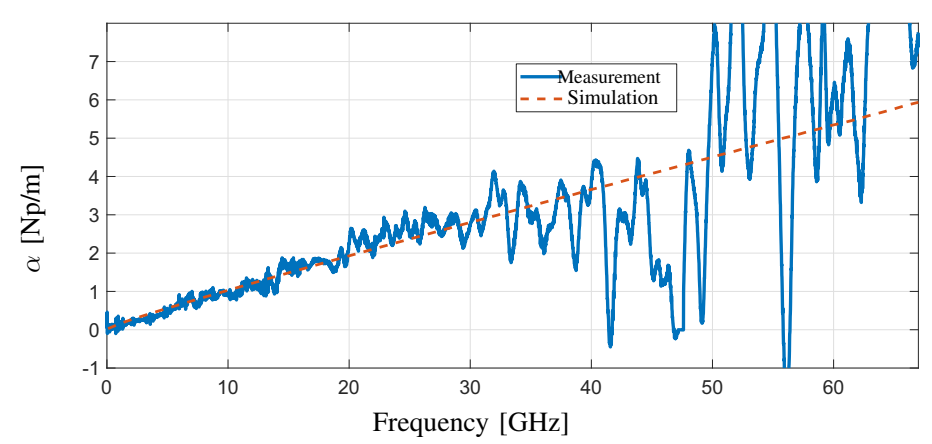

(a)

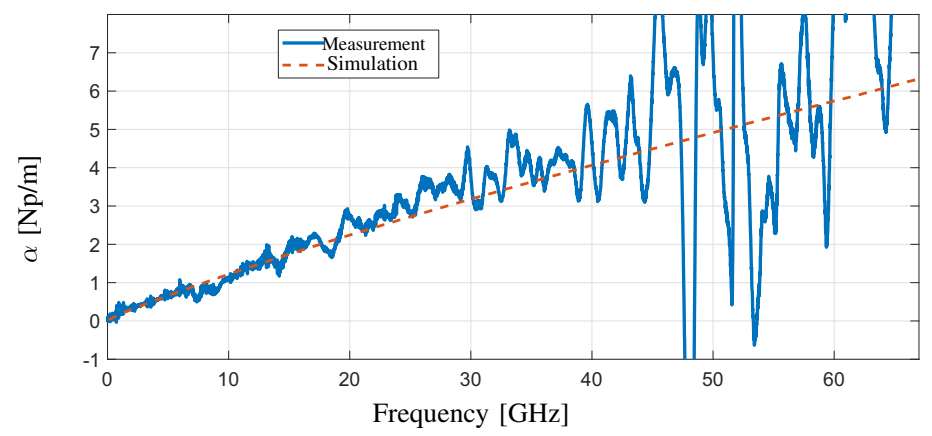

(c)

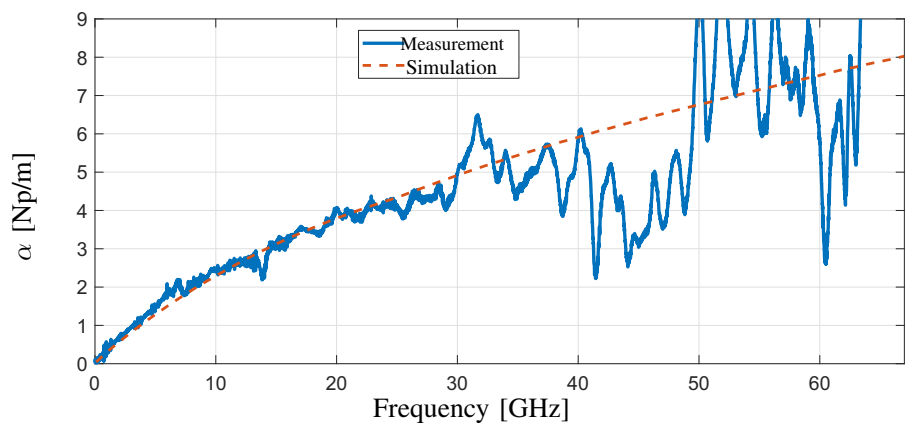

(e)

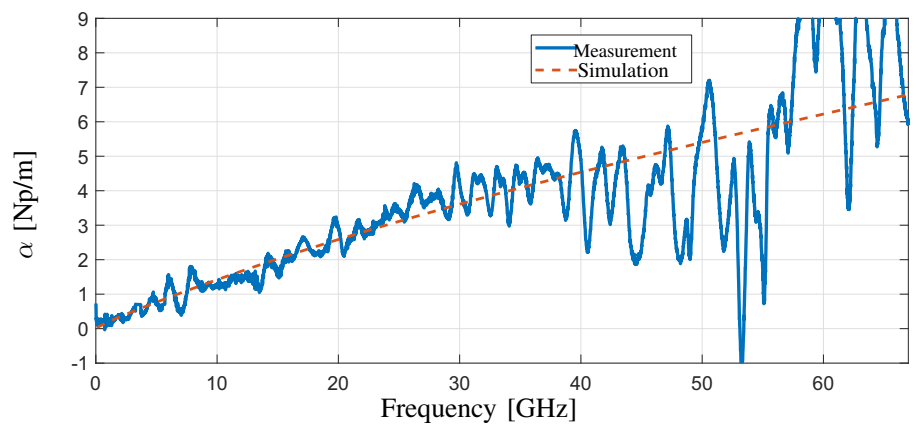

(g)

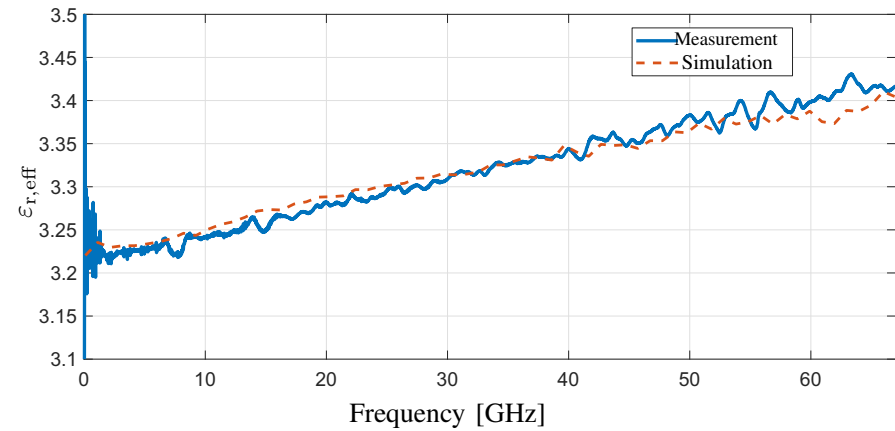

(b)

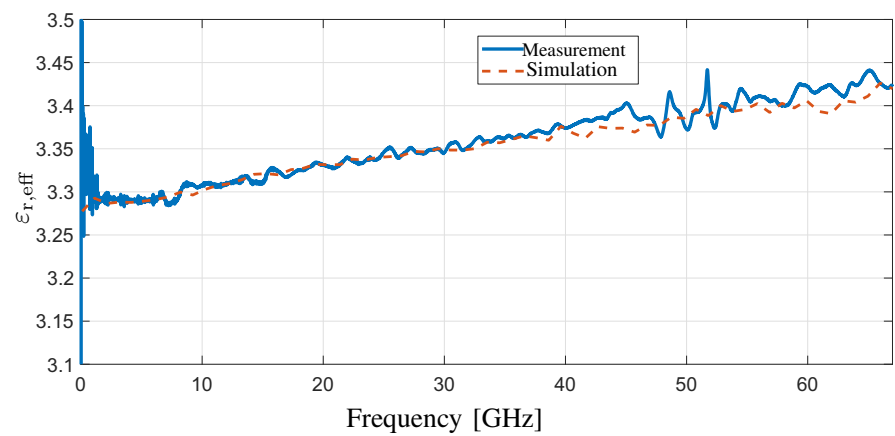

(d)

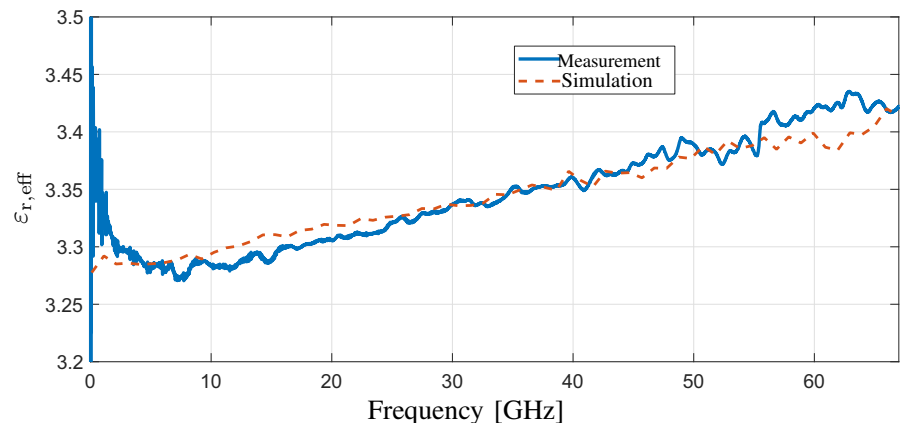

(f)

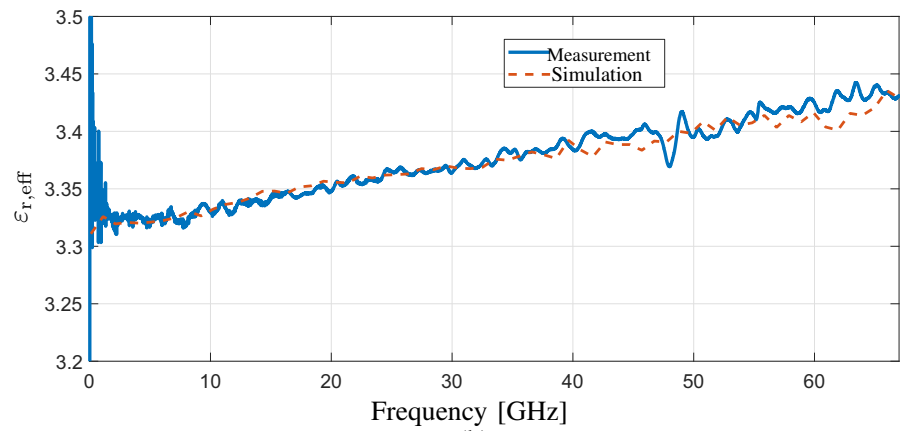

(h)

Fig. 7. Experimental results of the propagation constant using the proposed method with 7 line lengths in comparison with electromagnetic simulation results using: (a),(b) HIPS, (c),(d) ABS, (e),(f) TPU, (g),(h) CPE.

between measurements and simulations are achieved by using the developed model.

Figures 7 and 8 show the measured and simulated propagation constants in terms of attenuation constant and effective dielectric permittivity. For simplicity, considering that both pieces of ABS have similar results, only the one made by Ultimaker 3 has been depicted. As seen, an excellent agreement between measurements and simulation is achieved, highlighting the good accuracy in the phase constant and a good estimation of the loss tangent. Uncertainty in the measurement of the attenuation constant at high frequencies is due to the high values of the phase constant. Therefore, the errors are located in the attenuation constant, whose order of magnitude is much smaller.

Losses play a very important role in the design of structures for high frequency applications. Although these methods are 


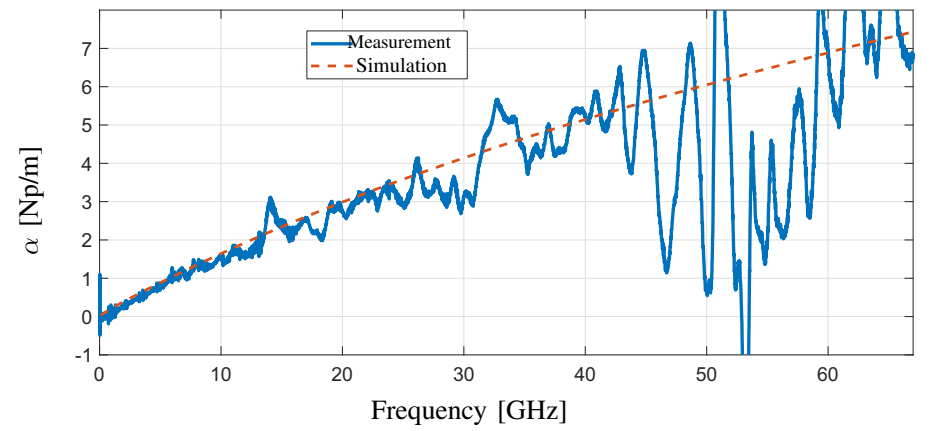

(i)

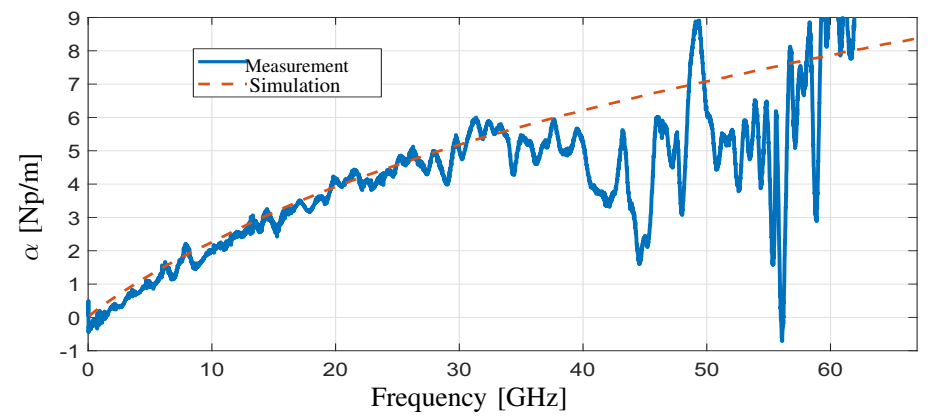

(k)

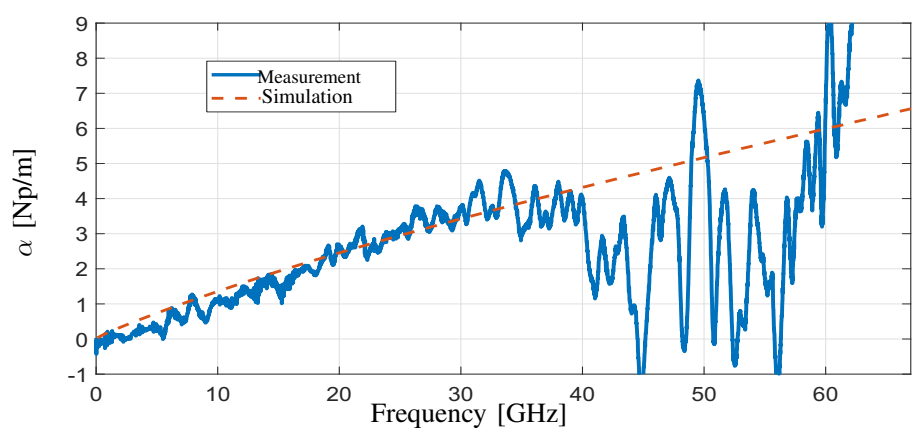

(m)

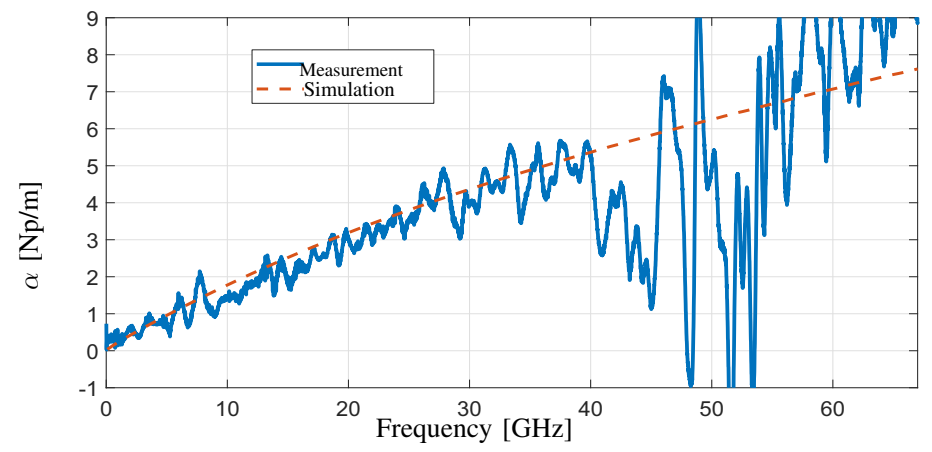

(o)

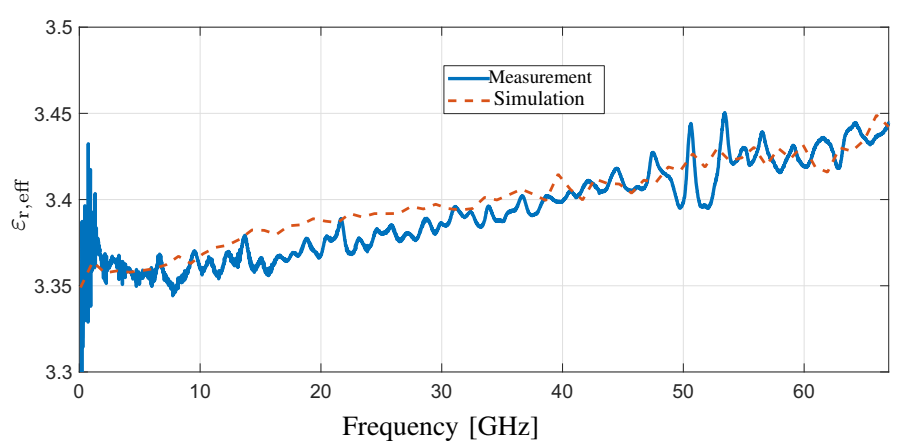

(j)

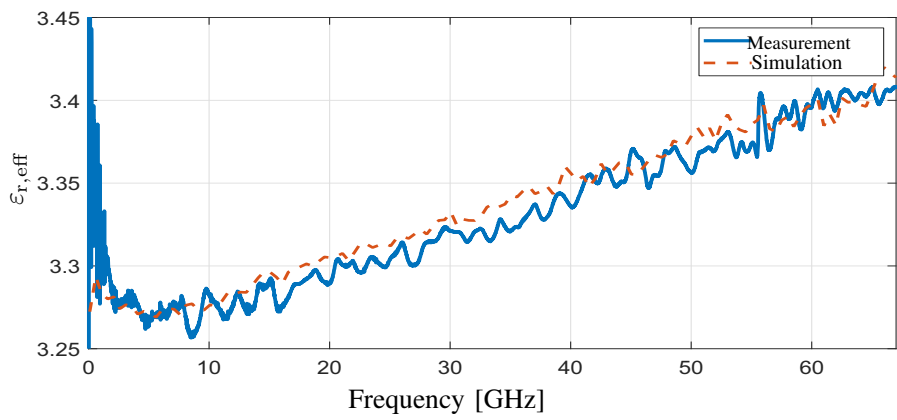

(1)

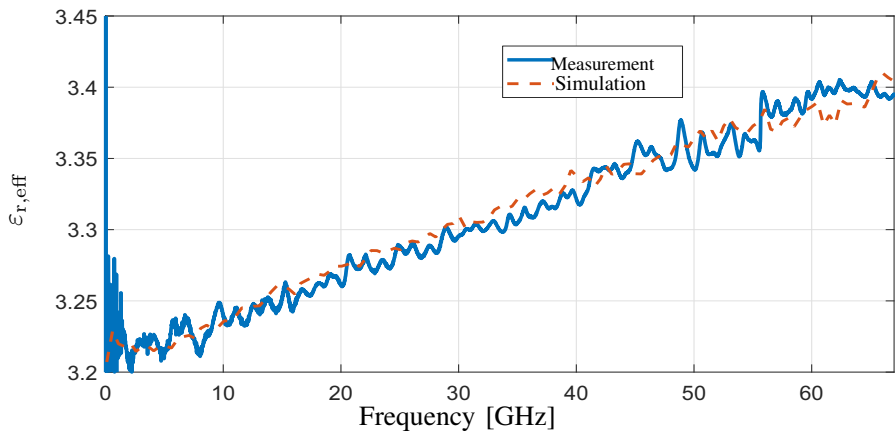

(n)

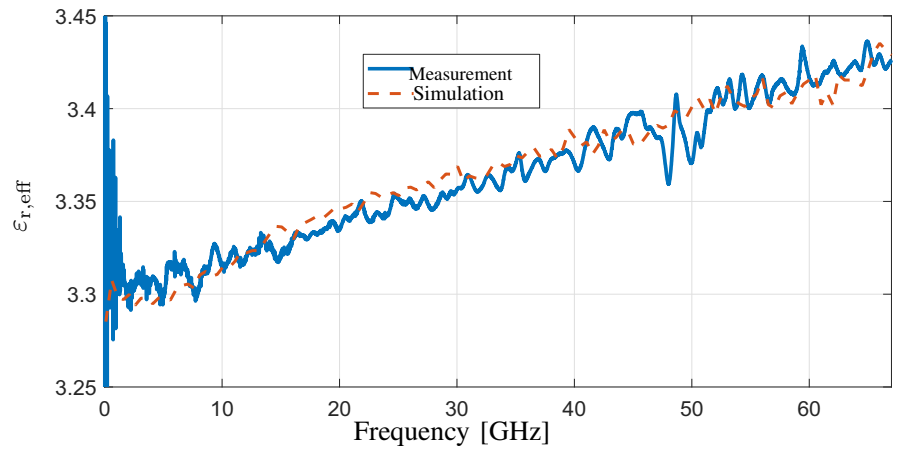

(p)

Fig. 8. Experimental results of the propagation constant using the proposed method with 7 line lengths in comparison with electromagnetic simulation results using: (i),(j) NYLON, (k), (l) FLEX, (m), (n) PLA, (o),(p) PETG.

not suitable for loss characterization, the proposed overestimation and the technique to select the most appropriate line lengths allow to obtain coherent results up to $40 \mathrm{GHz}$, where fluctuations of the attenuation constant limit the available frequency range of this method. However, this frequency range is quite wider and the obtained attenuation constant is more accurate than other in the state-of-the-art [11]. As it can be seen in Table I, HIPS has a low loss tangent, $\tan \delta=0.005$, and a low relative permittivity, $\varepsilon_{\mathrm{r}}=2.45$, if it is compared to the rest of substrates under test in this paper. HIPS is used in numerous applications because of its easy processing, performance and low cost [42]. The values of parameters estimated in this paper strongly agree with published data by manufacturers [43]. This fact makes HIPS a very good material 
to build support structures for circuits and antennas [22].

\section{CONCLUSION}

In this work, a simple technique to determine the broadband electromagnetic characteristics of material samples has been developed. It is very useful because of its simplicity and can be applied to both microstrip lines and lines covered with printing materials. No calibration is needed, and just several the repeatability of the transitions will strongly influence the results. Furthermore, the method allows to obtain very accurate results in terms of attenuation constant and effective dielectric permittivity by using only a few lines, whose length is optimized in order to minimize errors provoked by resonances. The achieved results show an excellent agreement between measurements and simulations, which is the reason why the proposed procedure can be considered as a good alternative to those of the state-of-the-art, due to its simplicity and the accuracy of the obtained results. HIPS has demonstrated excellent losses and can be established as a candidate for fabrication of electromagnetic structures due to its loss tangent close to 0.004 .

The proposed method can be used for any problem with transmission lines or waveguides. Despite having used in this article an indirect characterization process based on the measurement of the S-parameters of a multilayer structure, it has been possible to achieve models that include the substrate's dispersion or metal surface roughness. However, it is important to mention that one has to be careful with the gap between both substrates, to ensure that the error made is very small in the relative permittivity obtained. In addition, it must be considered that the characterization carried out will take into account the characteristics of the printed material as it is, with its infill, its possible printing defects, or its manufacturing temperatures. Therefore, if any of these parameters are changed, it would be convenient to carry out a new characterization.

\section{ACKNOWLEDGMENT}

The authors would like to thank Mario Mudarra, from M3DK Print, for the help given by printing several substrates during the COVID-19 lockdown.

\section{REFERENCES}

[1] E. Macdonald, R. Salas, D. Espalin, M. Perez, E. Aguilera, D. Muse, and R. B. Wicker, "3d printing for the rapid prototyping of structural electronics," IEEE Access, vol. 2, pp. 234-242, Dec 2014.

[2] P. I. Deffenbaugh, T. M. Weller, and K. H. Church, "Fabrication and microwave characterization of 3-d printed transmission lines," IEEE Microwave and Wireless Components Letters, vol. 25, no. 12, pp. 823825, Dec 2015.

[3] M. M. Abdin, J. Castro, J. Wang, and T. Weller, "Miniaturized 3d printed balun using high-k composites," in 2015 IEEE 16th Annual Wireless and Microwave Technology Conference (WAMICON), April 2015, pp. 1-3.

[4] A. Jones, S. Lucyszyn, E. Márquez-Segura, N. Ridler, J. Skinner, and D. Stokes, "3-d printed primary standards for calibration of microwave network analysers," Measurement, vol. 158, p. 107682, 2020. [Online]. Available: http://www.sciencedirect.com/science/article/pii/S0263224120302207

[5] P. I. Deffenbaugh, R. C. Rumpf, and K. H. Church, "Broadband microwave frequency characterization of 3-d printed materials," IEEE Transactions on Components, Packaging and Manufacturing Technology, vol. 3, no. 12, pp. 2147-2155, Dec 2013.
[6] V. Rizzoli, "Resonance measurement of single- and coupled-microstrip propagation constants," IEEE Transactions on Microwave Theory and Techniques, vol. 25, no. 2, pp. 113-120, Feb 1977.

[7] J. Sheen, "Comparisons of microwave dielectric property measurements by transmission/reflection techniques and resonance techniques," Meas. Sci. Technol, vol. 20, pp. 42 001-12, 042009.

[8] S. Moscato, R. Bahr, T. Le, M. Pasian, M. Bozzi, L. Perregrini, and M. M. Tentzeris, "Infill-dependent 3-d-printed material based on ninjaflex filament for antenna applications," IEEE Antennas and Wireless Propagation Letters, vol. 15, pp. 1506-1509, 2016.

[9] L. Catarinucci, R. Colella, P. Coppola, and L. Tarricone, "Microwave characterisation of polylactic acid for 3d-printed dielectrically controlled substrates," IET Microwaves, Antennas Propagation, vol. 11, no. 14, pp. 1970-1976, 2017.

[10] B. Bianco and M. Parodi, "Measurement of the effective relative permittivities of microstrip," Electronics Letters, vol. 11, no. 3, pp. 7172, February 1975.

[11] A. Hernández-Escobar, E. Abdo-Sánchez, J. Esteban, T. M. MartínGuerrero, and C. Camacho-Peñalosa, "Broadband determination of the even- and odd-mode propagation constants of coupled lines based on two-port measurements," IEEE Transactions on Microwave Theory and Techniques, vol. 68, no. 2, pp. 648-654, Feb 2020.

[12] G. A. Ramírez Arroyave and J. L. Araque Quijano, "Broadband characterization of $3 \mathrm{~d}$ printed samples with graded permittivity," in 2018 International Conference on Electromagnetics in Advanced Applications (ICEAA), Sep. 2018, pp. 584-588.

[13] A. C. Paolella, C. Corey, D. Foster, J. Desjardins, C. Smith, and L. Walters, "Broadband millimeter wave characterization of 3-d printed materials," in 2018 IEEE/MTT-S International Microwave Symposium IMS, June 2018, pp. 1565-1568.

[14] K. Szostak and P. Słobodzian, "Broadband dielectric measurement of pcb and substrate materials by means of a microstrip line of adjustable width," IEEE Microwave and Wireless Components Letters, vol. 28, no. 10 , pp. 945-947, 2018.

[15] A. M. Nicolson and G. F. Ross, "Measurement of the intrinsic properties of materials by time-domain techniques," IEEE Transactions on Instrumentation and Measurement, vol. 19, no. 4, pp. 377-382, 1970.

[16] W. B. Weir, "Automatic measurement of complex dielectric constant and permeability at microwave frequencies," Proceedings of the IEEE, vol. 62, no. 1, pp. 33-36, 1974.

[17] R. B. Marks, "A multiline method of network analyzer calibration," IEEE Transactions on Microwave Theory and Techniques, vol. 39, no. 7, pp 1205-1215, 1991

[18] D. F. Williams, C. M. Wang, and U. Arz, "An optimal multiline trl calibration algorithm," in IEEE MTT-S International Microwave Symposium Digest, 2003, vol. 3, 2003, pp. 1819-1822 vol.3.

[19] K. Andersson and C. Fager, "Statistical estimation of the propagation constant in multiline calibrations," in 2006 67th ARFTG Conference, 2006, pp. 133-136.

[20] B. T. W. Gillatt, M. D'Auria, W. J. Otter, N. M. Ridler, and S. Lucyszyn, "3-d printed variable phase shifter," IEEE Microwave and Wireless Components Letters, vol. 26, no. 10, pp. 822-824, 2016.

[21] S. Lucyszyn, X. Shang, W. J. Otter, C. W. Myant, R. Cheng, and N. M. Ridler, "Polymer-based 3d printed millimeter-wave components for spacecraft payloads," in 2018 IEEE MTT-S International Microwave Workshop Series on Advanced Materials and Processes for $\mathrm{RF}$ and $\mathrm{THz}$ Applications (IMWS-AMP), 2018, pp. 1-3.

[22] E. García-Marín, E. Márquez-Segura, P. Sánchez-Olivares, J. L. MasaCampos, J. A. Ruiz-Cruz, and C. Camacho-Peñalosa, "Ink-jet implementation of stacked-patch antenna for wireless applications," in 2019 IEEE MTT-S International Microwave Workshop Series on Advanced Materials and Processes for RF and THz Applications (IMWS-AMP), 2019, pp. 151-153.

[23] M. A. Couker and L. J. Kushner, "A microstrip phase-trim device using a dielectric overlay," IEEE Transactions on Microwave Theory and Techniques, vol. 42, no. 11, pp. 2023-2026, Nov 1994.

[24] G. F. Engen and C. A. Hoer, "Thru-reflect-line: An improved technique for calibrating the dual six-port automatic network analyzer," IEEE Transactions on Microwave Theory and Techniques, vol. 27, no. 12, pp. 987-993, 1979.

[25] R. A. Soares, P. Gouzien, P. Legaud, and G. Follot, "A unified mathematical approach to two-port calibration techniques and some applications," IEEE Transactions on Microwave Theory and Techniques, vol. 37, no. 11 , pp. 1669-1674, 1989.

[26] R. R. Pantoja, M. J. Howes, J. R. Richardson, and R. D. Pollard, "Improved calibration and measurement of the scattering parameters of 
microwave integrated circuits," IEEE Transactions on Microwave Theory and Techniques, vol. 37, no. 11, pp. 1675-1680, 1989.

[27] Moon-Que Lee and Sangwook Nam, "An accurate broadband measurement of substrate dielectric constant," IEEE Microwave and Guided Wave Letters, vol. 6, no. 4, pp. 168-170, 1996.

[28] J. van Heuven and T. Rozzi, "The invariance properties of a multivalue n-port in a linear embedding," IEEE Transactions on Circuit Theory, vol. 19 , no. 2, pp. 176-183, 1972.

[29] J. A. Reynoso-Hernandez, "Reliable method for computing the phase shift of multiline $1 \mathrm{rl}$ calibration technique," IEEE Microwave and Wireless Components Letters, vol. 12, no. 10, pp. 395-397, 2002.

[30] M. Wojnowski, V. Issakov, G. Sommer, and R. Weigel, "Multimode trl calibration technique for characterization of differential devices," IEEE Transactions on Microwave Theory and Techniques, vol. 60, no. 7, pp. 2220-2247, 2012.

[31] M. Kirschning and R. H. Jansen, "Accurate model for effective dielectric constant of microstrip with validity up to millimetre-wave frequencies," Electronics Letters, vol. 18, no. 6, pp. 272-273, March 1982.

[32] "Ro4000 series high frequency circuit materials," https://rogerscorp.com/-/media/project/rogerscorp/documents/advancedconnectivity-solutions/english/data-sheets/ro4000-laminates-ro4003cand-ro4350b-data-sheet.pdf.

[33] M. Barbuto, A. Alù, F. Bilotti, and A. Toscano, "Characteristic impedance of a microstrip line with a dielectric overlay," COMPEL: Int $J$ for Computation and Maths. in Electrical and Electronic Eng., vol. 32, 112013.

[34] E. Hammerstad and O. Jensen, "Accurate models for microstrip computer-aided design," in 1980 IEEE MTT-S International Microwave symposium Digest, May 1980, pp. 407-409.

[35] E. Y. Terán-Bahena, S. C. Sejas-García, and R. Torres-Torres, "Permittivity determination considering the metal surface roughness effect on the microstrip line series inductance and shunt capacitance," IEEE Transactions on Microwave Theory and Techniques, vol. 68, no. 6, pp. 2428-2434, 2020.

[36] A. K. Verma, Nasimuddin, and E. K. Sharma, "Analysis and circuit model of a multilayer semiconductor slow-wave microstrip line," IEE Proceedings - Microwaves, Antennas and Propagation, vol. 151, no. 5, pp. 441-449, 2004.

[37] "Copper foils for high frequency materials," www.rogerscorp.cn/documents/749/acs/Copper-Foils-for-HighFrequency-Circuit-Materials.pdf.

[38] S. Groiss, I. Bardi, O. Biro, K. Preis, and K. R. Richter, "Parameters of lossy cavity resonators calculated by the finite element method," IEEE Transactions on Magnetics, vol. 32, no. 3, pp. 894-897, 1996.

[39] S. Kim and J. Baker-Jarvis, "An approximate approach to determining the permittivity and permeability near $\lambda / 2$ resonances in transmission/reflection measurements," Progress In Electromagnetics Research $B$, vol. 58, pp. 95-109, 012014

[40] N. Abdul Malek, A. Mohd Ramly, A. Sidek, and S. Mohamad, "Characterization of acrylonitrile butadiene styrene for $3 \mathrm{~d}$ printed patch antenna," Indonesian Journal of Electrical Engineering and Computer Science, vol. 6, p. 116, 042017.

[41] C. Dichtl, P. Sippel, and S. Krohns, "Dielectric properties of 3d printed polylactic acid," Advances in Materials Science and Engineering, vol. 2017, pp. 1-10, 072017.

[42] M. Gauthier, Modern Styrenic Polymers: Polystyrenes and Styrenic Copolymers. ASM International, 1995.

[43] J. Scheirs and D. Priddy, Modern Styrenic Polymers: Polystyrenes and Styrenic Copolymers. John Wiley and Sons, 2003.

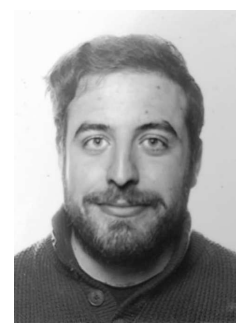

Mario Pérez-Escribano (S'19) was born in Alcalá la Real, Jaén, Spain, in December 1993. He received a B.S. and a M.S. degrees in telecommunication engineering, from the University of Málaga, Málaga, Spain and from the University Carlos III de Madrid, Madrid, Spain, in 2015 and 2017, respectively.

In 2014, he joined the Departamento de Ingeniería de Comunicaciones, Escuela Técnica Superior de Ingeniería (E.T.S.I.) de Telecomunicación, Universidad de Málaga, Málaga, Spain, where he is currently pursuing a $\mathrm{PhD}$ degree. His research interests are focused on the analysis of structures made by additive manufacturing techniques, planar circuits and dielectric resonator antennas.

Mr. Pérez-Escribano is the recipent of a Spanish Ministry of Education, Culture and Sports Scholarship (2017-2021).

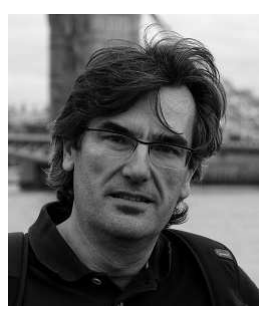

Enrique Márquez-Segura (S'93--M'95--SM'06) was born in Málaga, Spain, in April 1970. He received the Ingeniero de Telecomunicación and Doctor Ingeniero degrees from the Universidad de Málaga, Málaga, Spain, in 1993 and 1998, respectively.

In 1994, he joined the Departamento de Ingeniería de Comunicaciones, Escuela Técnica Superior de Ingeniería (E.T.S.I.) de Telecomunicación, Universidad de Málaga, Málaga, Spain, where, in 2001, he became an Associate Professor. His current research interests include electromagnetic material characterization, additive manufacturing, measurement techniques, and RF, microwave and millimeter wave circuits design for communication applications.

Dr. Márquez-Segura was the recipient of a Spanish Ministry of Education and Science Scholarship (1994-1995). 